\title{
Double Shock Control Bump Design Optimization Using Hybridised Evolutionary Algorithms
}

\author{
DongSeop Lee, Jacques Periaux, Jordi Pons-Prats, Gabriel Bugeda and Eugenio Oñate
}

\begin{abstract}
The paper investigates two advanced optimisation methods for solving active flow control device shape design problem and also compares their optimisation efficiency in terms of computational cost and design quality. The first optimisation method uses Hierarchical Asynchronous Parallel Multi-Objective Evolutionary Algorithm (HAPMOEA) and the second uses Hybridized EA with Nash-Game strategies. Both optimisation method are based on a canonical evolution strategy and incorporates the concepts of parallel computing and asynchronous evaluation. For the practical test case, one of active flow control devices named Shock Control Bump (SCB) is considered and it is applied to Natural Laminar Flow (NLF) aerofoil. The concept of SCB is to decelerate supersonic flow on upper/lower surface of transonic aerofoil that leads delay of shock occurrence. Such active flow technique reduces a total drag at transonic speeds.

Numerical results clearly show that Hybrid-Game helps EA to accelerate optimisation process, and also applying SCB on the suction and pressure sides significantly reduces transonic wave drag and improves lift on drag $(L / D)$ value when compared to the baseline design.
\end{abstract}

Keywords - Active Flow Control, Shock Control Bump, Shape design optimisation, Hybrid-Game, Nash Equilibrium, Evolutionary Algorithm.

\section{INTRODUCTION}

$\mathrm{D}$ EVELOPING efficient optimisation techniques is the most challenging task in the field of Evolutionary Algorithms (EAs) research due to the complexity of modern design problems. One of emerging techniques to improve an optimisation performance can be the use of Nash-equilibrium concept which will be acting as a pre-conditioner of global

DongSeop Lee is with the International Center for Numerical Methods in Engineering (CIMNE), Room 216, Parque Mediterraneo de la Tecnologia (PMT) Edificio C3 CIMNE, C/Esteve Terrdas n5 08860 Castelldefels Spain. (phone: +34 934010 807; fax: +34 934137 242; e-mail: ds.chris.lee@gmail.com).

Jacques Periaux is with the International Center for Numerical Methods in Engineering (CIMNE) as a UNESCO chair, Barcelona, Spain. (e-mail: iperiaux@gmail.com).

Jordi Pons-Prats is with the International Center for Numerical Methods in Engineering (CIMNE) Barcelona, Spain. (e-mail: jpons@cimne.upc.edu).

Gabriel Bugeda is with the Universitat Politècnica de Catalunya (UPC) and the International Center for Numerical Methods in engineering (CIMNE). (e-mail: bugeda@cimne.upc.edu).

Eugenio Onate is with the International Center for Numerical Methods in Engineering (CIMNE) as a director, Barcelona Spain. (e-mail: onate@cimne.upc.edu). optimizer.

Lee et al. [1] studied the concept of Hybrid-Game (Pareto + Nash) coupled to a well-known MOEA; Non-dominating Sort Genetic Algorithm II (NSGA-II) [2] to solve Unmanned Aerial System (UAS) multi-objective Mission Path Planning System (MPPS) design problems where the Hybrid-Game improves the NSGA-II performance by $80 \%$ when compared to the original NSGA-II. In addition, Lee et al. [3] hybridised NSGA-II with Nash-Game strategy to study a role of Nash-Players in Hybrid-Game by solving multi-objective mathematical design problems convex, discontinuous, etc. And Hierarchical Asynchronous Parallel Multi-Objective Evolutionary Algorithm (HAPMOEA) [4] is also hybridised to solve a real-world robust multidisciplinary design problem. Numerical results show that the Hybrid-Game improves $70 \%$ of HAPMOEA performance while producing better Pareto optimal solutions. References [1, 3, 5] clearly describes merits of using Hybrid-Game coupled to MOEA for engineering design applications which consider a complex geometry or a large number of design variables. Hybrid-Game has two major characteristics; the first is a decomposition of design problem, for instance, a multi-objective design problem will be split into several simpler single-objective problems corresponding to Nash-Players which have their own design search space. The second is that Nash-Players are synchronised with Global/Pareto-Player as a pre-conditioner hence Pareto-Player can accelerate the optimisation process by using a set of elite designs obtained by the Nash-Players during optimisation.

The main goal of this paper is to investigate an efficiency of Hybrid-Game for a single-objective design problem. The search space herein will be decomposed respect to Nash-Players. In this paper, HAPMOEA is hybridized with Nash game strategy to improve optimisation efficiency. Both optimisation methods are implemented to Active Flow Control (AFC) device shape design optimisation and their performance are compared in terms of computational cost and design quality.

Recent advances in design tools, materials, electronics and actuators offer implementation of flow control technologies to improve aerodynamic efficiency [6 -10]. Such aerodynamic improvement saves mission operating cost while condensing critical aircraft emissions. The main benefits of using ACF 
techniques on current transonic aircraft are the improvement of the aerodynamic efficiency and the reduction of the manufacturing cost when compared to designing new airfoil or wing planform shape.

In this paper, one of active flow control devices; double Shock Control Bump (SCB) [8 -10] on the suction and pressure sides of Natural Laminar Flow (NLF) aerofoil; RAE 5243 [10, 11 is investigated in order to reduce a transonic total drag, especially a wave drag at the critical flight conditions where two shocks occur.

The rest of paper is organised as follows; Section II describes optimisation method. Aerodynamic analysis tools are demonstrated and validated at Section III. Section IV demonstrates the use of SCB. Section V considers double SCB design optimisation using HAPMOEA and Hybrid-Game. Section VI delivers conclusion and future works.

\section{OPtIMISATION METHODS}

The evolutionary algorithm used in this paper is based on Covariance Matrix Adaptation Evolutionary Strategies (CMA-ES) [12, 13] which incorporates an asynchronous parallel computation and a Pareto tournament selection [14 -16]. The first method; HAPMOEA uses the concept of hierarchical multi-population topology which can handle different models including precise, intermediate and approximate models. Each node (Node0 Node6) belonging to the different hierarchical layer can be handled by a different EA code. The second method hybridises HAPMOEA by applying a concept of Nash-Equilibrium instead of the concept of hierarchical multi-population topology [4, 17]. The Nash-Game players choose their own strategy to improve their own objective. Both HAPMOEA and Hybridised EA are coupled to the aerodynamic analysis tool. Details and validations of HAPMOEA and Hybrid-Game can be found in references [1, 3, 17].

\section{AERODYNAMIC ANALYSIS TOOLS}

In this paper, Euler + Boundary layer: MSES written by Drela [18] is utilised. The MSES software is a coupled viscous/inviscid Euler method for the analysis and design of multi-element/single-element aerofoils. It is based on a streamline-based Euler discretization and a two-equation integral boundary layer formulation coupled through the displacement thickness and solved simultaneously by a full Newton method. To obtain a prescribed lift coefficient $C_{l}$, the angle of attack $(\alpha)$ of the aerofoil is adapted or the other way around. Figure 1 a) shows the mesh $(36 \times 213)$ obtained by MSES that will be applied to optimisation.

The validation test is conducted on $4 \times 2.8 \mathrm{GHz}$ CPU. The flight conditions are Mach number $\left(M_{\infty}\right)=0.729$, angle of attack $(\alpha)=2.31^{\circ}$ and Reynolds number $(\operatorname{Re})=6.5 \times 10^{6}$. Figure $1 \mathrm{~b})$ compares the results obtained by MSES to the wind tunnel experimental data [19]. It can be seen there is good agreement between $C p$ distributions obtained by MSES and the wind tunnel data.

\section{WAVE DRAg REDUCTION VIA SHOCK CONTROL BUMP}

At transonic speed, the flow over the high camber wing causes shock waves where there is a large amount of gas property changes and the flow becomes irreversible.

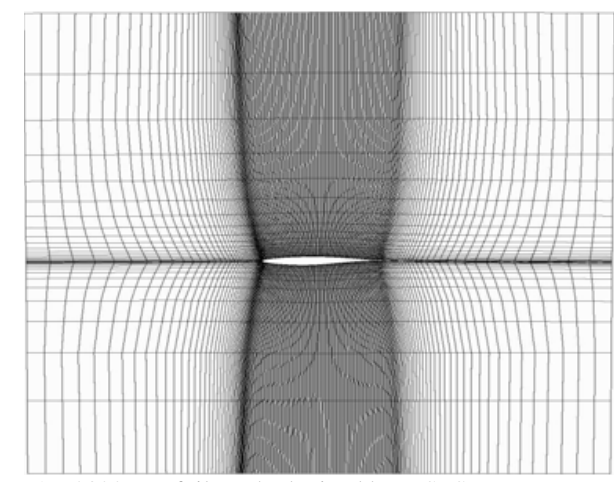

Fig. 1 a). RAE 2822 aerofoil mesh obtained by MSES.

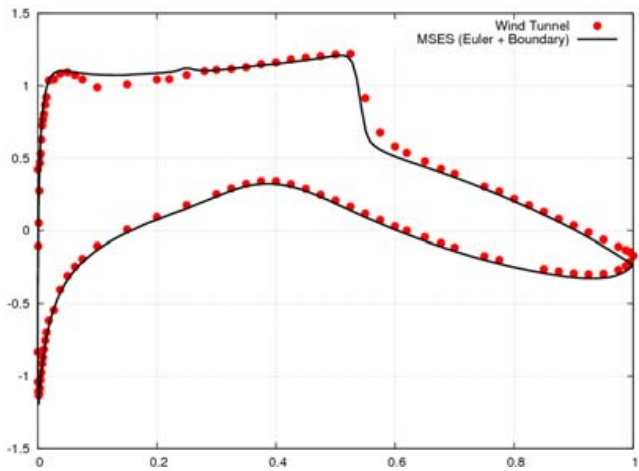

Fig. 1 b). Comparison of $C p$ distributions obtained by MSES (line) and wind tunnel experimental data (dots).

Through the shock, total pressure decreases and entropy increases which means there is a loss of energy. In other words, there is an increment of wave drag. To cope with this problem, Ashill et al. 1992 [7] proposed the concept of a transonic bump which so-called Shock Control Bump (SCB) by using geometry adaption on an aerofoil. As illustrated in Figure 2, the typical design variables for SCB are: length, height and peak position and, the center of SCB will be located at sonic point where the flow speed transits from supersonic to subsonic on the transonic aerofoil design.



Fig. 2. Design components of Shock Control Bump.

Figure 3 illustrates the concept and benefit of using SCB. The transonic flow over normal aerofoil without SCB accelerates to supersonic and the pressure forms a strong shock 
that leads a high wave drag $\left(C d_{\text {Wave }}\right)$. However, the pressure difference over the SCB causes a deceleration of supersonic flow which delays shock occurrence. SCB cannot totally remove a shock, but it produces a weaker shock or breaks into isentropic compression waves (lower $C d_{\text {Wave }}$ ).

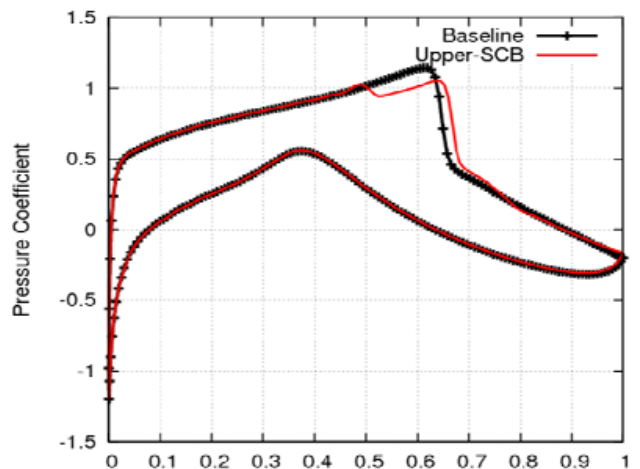

Fig. 3. $C p$ distributions obtained by RAE 2822 (dots and line) and with SCB (line).

Table I compares the aerodynamic performance obtained by RAE 2822 and with SCB. Even though applying SCB on RAE 2822 produces $5 \%$ higher viscous drag $\left(\Delta C d_{\text {Viscous }}=0.0005\right)$, it reduces $60 \%$ wave drag $\left(\Delta C d_{\text {Wave }}=0.0036\right)$ while improving $19 \%$ of $L / D$ when compared to RAE 2822 aerofoil.

TABLE I

AERODYNAMIC CHARACTERISTICS

\begin{tabular}{|c|c|c|c|c|}
\hline Aerofoil & $C d_{\text {Total }}$ & $\bar{C} C d_{\text {Viscous }}$ & $\bar{C} C d_{\text {Wave }}$ & $L / D$ \\
\hline RAE 2822 & 0.0153 & 0.0093 & 0.0060 & 34.34 \\
\hline with SCB & $0.0123(-20 \%)$ & $0.0098(+5 \%)$ & $0.0024(-60 \%)$ & $42.6(+24 \%)$ \\
\hline
\end{tabular}

Applying SCB on either upper or lower surface of aerofoil will produces slightly thicker thickness ratio $(t / c)$ which causes increment of viscous drag $\left(C d_{V i s c o u s}\right)$ however the use of SCB is still beneficial due to $C d_{\text {Wave }}$ reduction especially when the Mach number is higher than critical Mach number where the shock starts appearing.

In Section V, the shape of SCB is optimized at critical flight conditions where two shocks occur on the suction and pressure sides of aerofoil. This flight conditions make a suitable application for Hybrid-Game (Global + Nash) since two SCBs are required. The aerodynamic characteristics of baseline with optimal double SCB are also investigated at normal flight conditions where a single shock is on the upper surface of aerofoil.

\section{SCB DESIGN OPTIMISATION ON RAE 5243}

For baseline design, a natural laminar flow aerofoil RAE 5243 is selected as shown in Figure 4 a). The problem considers the critical flow conditions; $M_{\infty}=0.8, C_{l}=0.175, R e=18.63 \times$ $10^{6}$ where two shocks occur on the suction and pressure sides of RAE 5243 aerofoil as shown in Figure 4 b).

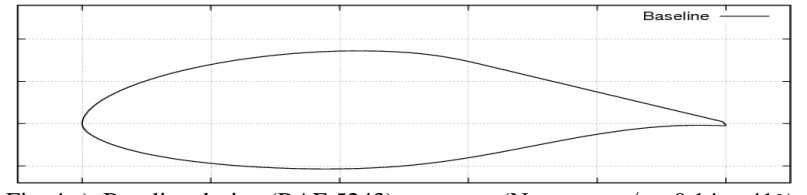

Fig. 4 a). Baseline design (RAE 5243) geometry (Note: $\max t / c=0.14$ at $41 \% c$ and max camber $=0.018$ at $54 \% \mathrm{c}$ ).

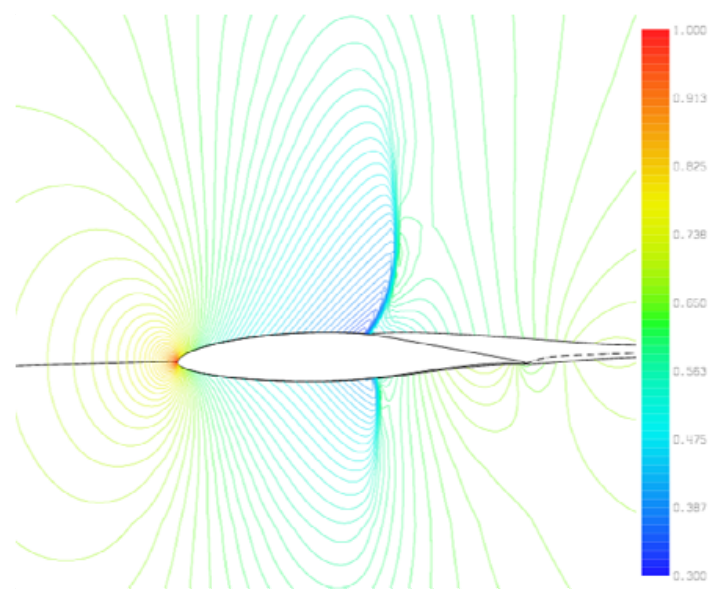

Fig. 4 b). $P / P_{0}$ contour of RAE 5243.

The upper and lower sonic points are occurred at $62.6 \%$ and $58.1 \%$ of chord respectively. In following sections, double SCB design optimization using HAPMOEA and Hybrid-Game are conducted to minimize the total drag $\left(C d_{\text {Total }}\right)$. The aerodynamic analysis tool; MSES will run two times at each function evaluation; the first run will analyse upper SCB and then upper and lower SCB will be analysed at the second run.

\section{A. Evaluation Mechanism for HAPMOEA and Hybrid-Game}

Figure 5 a) shows the evaluation mechanism for HAPMOEA which consists of hierarchical multi-population (Node 0 Node 6) based on multi-resolution. Each population will run aerodynamic analysis tool two times to evaluation double SCB design in different resolution conditions.
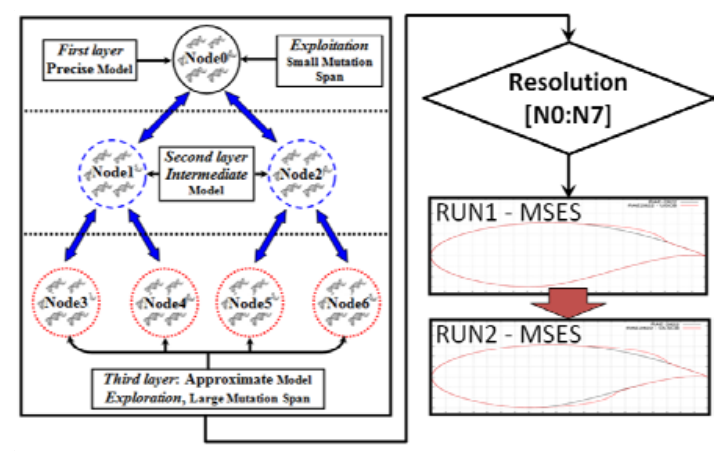

Fig. 5 a). Evaluation mechanism of HAPMOEA.

Figure 5 b) shows the evaluation mechanism for Hybrid-Game which employs three players; Global-Player and Nash-Player 1 and 2. Solely Global-Player runs aerodynamic analysis tool two times since its optimisation domain includes 
both upper and lower SCB. However, analysis tool will run only once for Nash-Player 1 and 2 due to the Nash-Game characteristics; decomposition of problem. For Hybrid-Game, double SCB design problem becomes two single SCB design problems; Nash-Game 1 will only optimize upper SCB with elite lower SCB obtained by Nash-Player 2 while Nash-Player 2 will optimise lower SCB with elite upper SCB design from Nash-Player 1 . The elite designs obtained by Nash-Players will be seed to the population of the Global-Player that will allow Global-Player to accelerate optimisation process.

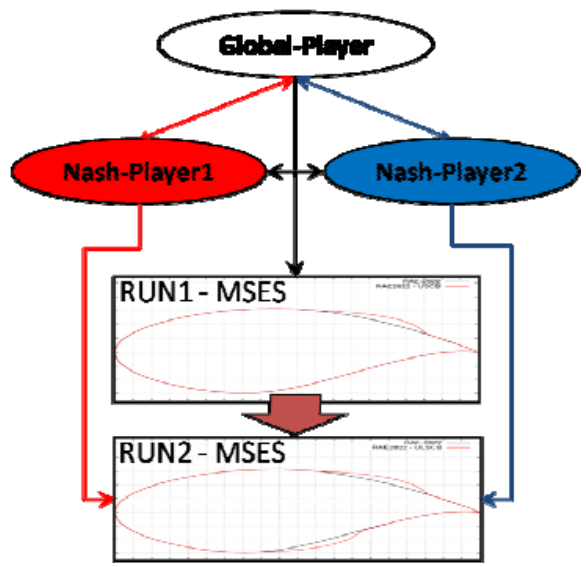

Fig. 5 b). Evaluation mechanism of Hybrid-Game.

\section{B. SCB Design Optimisation using HAPMOEA}

\section{Problem Definition}

This test case considers a single objective double SCB design optimisation using HAPMOEA to minimize total drag $\left(C d_{\text {Total }}\right)$ which consists of viscous drag $\left(C d_{V i s c o u s}\right)$ and wave drag $\left(C d_{\text {Total }}\right)$. The flow conditions are $M_{\infty}=0.8, C_{l}=0.175, R e=$ $18.63 \times 10^{6}$. The fitness function is shown in equation (1).

$$
f\left(U_{-} S C B, L_{-} S C B\right)=\min \left(C d_{\text {Total }}\right)
$$

where $C d_{\text {Total }}=C d_{\text {Viscous }}+C d_{\text {Wave }}$.

\section{Design Variables}

The design variable bounds for both SCB on the suction and pressure sides are illustrated in Table II. In total, six design variables are considered for double SCB.

TABLE II

SCB DESIGN VARIABLES AND BOUNDS

\begin{tabular}{ccc}
\hline \hline Design Variables & Lower bound & Upper bound \\
\hline Length (\% chord) & 15 & 30 \\
Height (\% chord) & 0.15 & 0.65 \\
Peak position & 0 & 100 \\
\hline \hline Note: Peak position is in \% of SCB length
\end{tabular}

The centre of SCB (50\% of SCB length) will be positioned where the flow speed transits from supersonic to subsonic.

\section{$\underline{\text { Implementation }}$}

The following conditions are for multi-resolution/population hierarchical populations.
- $1^{\text {st }}$ Layer: Population size of 10 with a computational grid of $36 \times 213$ points (Node 0 ).

- $2^{\text {nd }}$ Layer: Population size of 20 with a computational grid of $24 \times 131$ points (Node1, Node2).

- $3^{\text {rd }}$ Layer: Population size of 20 with a computational grid of $36 \times 111$ points (Node3 Node6).

Note: these grid conditions produce less than 5\% accuracy error compared to precise model at the $1^{\text {st }}$ layer (Node0).

\section{Numerical Results}

As illustrated in Figure 6, the algorithm was allowed to run for 24 hours and 2,508 function evaluations using single $4 \times 2.8$ $\mathrm{GHz}$ processor. The algorithm converged at 1,053 function evaluations with $C d_{\text {Total }}=0.03441$ after ten hours.

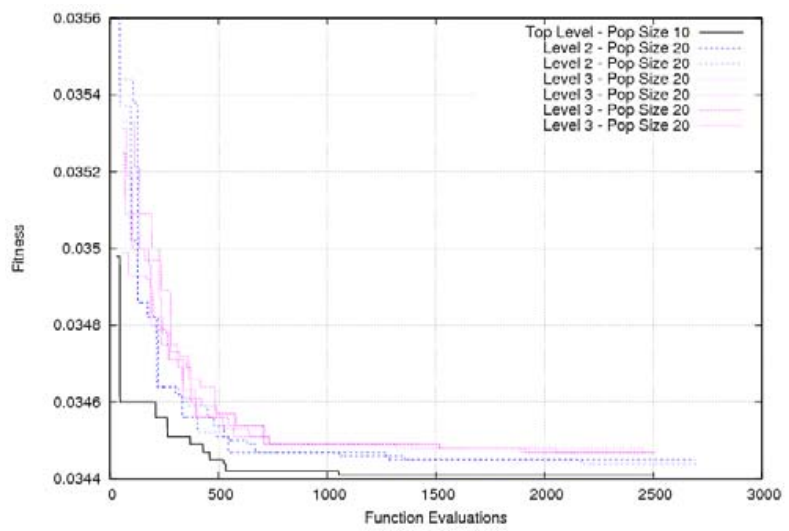

Fig. 6. Convergence objective using HAPMOEA.

Table III compares the aerodynamic characteristics obtained by the baseline design (RAE 5243) and the baseline design with upper and lower SCBs. Applying SCB on upper surface of RAE 5243 aerofoil saves the wave drag by $8 \%$ which leads $12 \%$ of total drag reduction. This optimal double SCB improves $L / D$ by $13.0 \%$.

TABLE III

\begin{tabular}{cccc}
\multicolumn{4}{c}{ AERODYNAMIC CHARACTERISTICS } \\
\hline \hline Aerofoil & $C d_{\text {Total }}$ & $C d_{\text {Wave }}$ & $L / D$ \\
\hline Baseline & 0.03898 & 0.0088 & 4.49 \\
with SCB & $0.03441(-12 \%)$ & $0.0081(-8 \%)$ & $5.08(+13 \%)$ \\
\hline \hline
\end{tabular}

Note: $C l$ is fixed to 0.175 .

TABLE IV

OPTIMAL DOUBLE SCB DESIGN COMPONENTS

\begin{tabular}{cccc}
\hline \hline Variables & Length $(\% c)$ & Height $(\% c)$ & Peak Position \\
\hline U_SCB & 23.31 & 0.649 & 84.95 \\
L_SCB & 26.38 & 0.477 & 75.98 \\
\hline \hline
\end{tabular}

Note: Peak position is in \% of SCB length. The U_SCB starts from $x$ and $y$ coordinates $(0.5084,0.0838)$ to $(0.7416,0.0480)$ and L_SCB is positioned from (0.4397, -0.05269$)$ to $(0.7035,-0.0258)$.

The optimal double SCB shape of is described in Table IV. Figure 7 compares the geometry of the baseline design and baseline with the optimal SCB which has same $t / c$ while the max camber is increased by 0.0005 and its position is moved $16 \%$ c towards to the trailing edge when compared to the baseline design. 


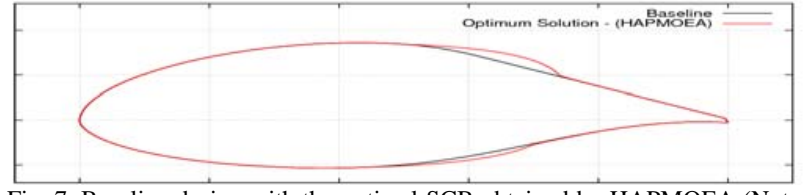

Fig. 7. Baseline design with the optimal SCB obtained by HAPMOEA (Note: $\max t / c=0.14$ at $41 \% c$ and $\max$ camber $=0.0209$ at $69.8 \% c$ ).

Figure 8 shows the contour of baseline design with the optimal double SCB. It can be seen that the strong shocks on the baseline design shown in Figure 4 b) get weaker by adding double SCB.

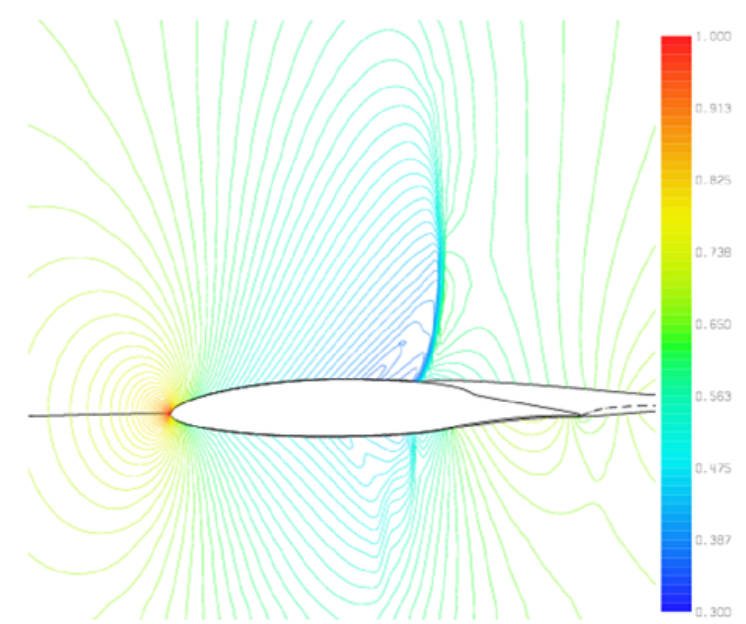

Fig. 8. $P / P_{0}$ contour of the optimal SCB solution obtained by HAPMOEA

Figure 9 compares $C p$ distribution obtained by the baseline design and the baseline design with upper and lower SCB. It can be seen that the total drag is reduced by $9 \%$ while the double SCB reduces $12 \%$ of total drag. The shock on upper surface is delayed while the shock on lower surface becomes weak isentropic waves.

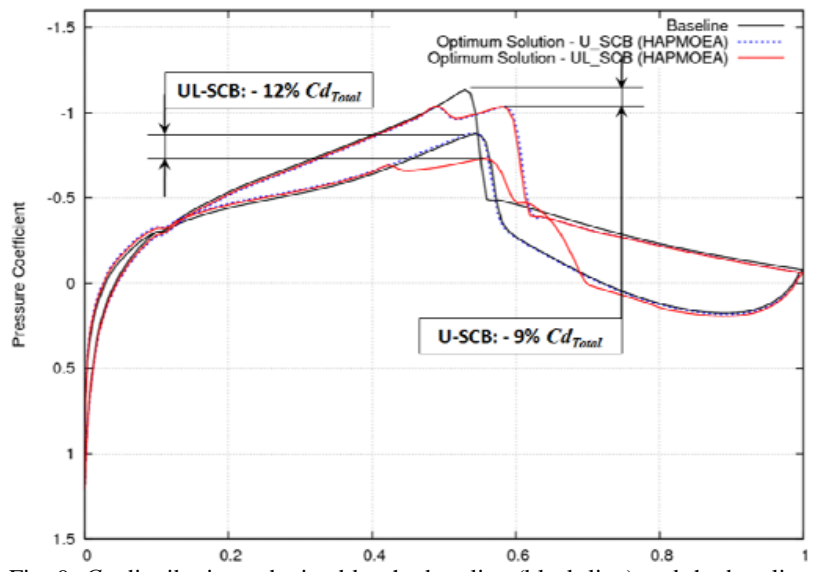

Fig. 9. $C p$ distributions obtained by the baseline (black line) and the baseline with the optimal double SCB solution (broken line: only upper SCB, red line: upper and lower SCBs).

\section{SCB Design Optimisation using Hybrid-Game}

\section{Problem Definition}

This test case considers a single objective double SCB design optimisation using Hybrid-Game on MOEA to minimize total drag at flow conditions $M_{\infty}=0.8, C_{l}=0.175, R e=18.63 \times 10^{6}$. Hybrid-Game consists of three players; one Global-Player $(G P)$, two Nash-Players (NP1 and NP2) instead of hierarchical multi-population/resolution (Node $0 \sim$ Node 6 ). The fitness functions for Hybrid-Game are shown in equation (2).

$$
\begin{gathered}
f_{G P}\left(U_{-} S C B, L_{-} S C B\right)=\min \left(C d_{\text {Total }}\right) \\
f_{N P 1}\left(U_{-} S C B, L_{-} S C B^{*}\right)=\min \left(C d_{\text {Total }}\right) \\
f_{N P 2}\left(U_{-} S C B^{*}, L_{-} S C B\right)=\min \left(C d_{\text {Total }}\right)
\end{gathered}
$$

where $C d_{\text {Total }}=C d_{\text {Viscous }}+C d_{\text {Wave }} . U_{-} S C B$ and $L \_S C B$ represent upper and lower SCB, and * is elite SCB design obtained by Nash-Players. $U_{-} S C B^{*}$ and $L_{-} S C B^{*}$ are elite SCB designs obtained by Nash-Player 1 and $\overline{2}$. These elite SCB designs will be seeded to the population of Global-Player at every 10 function evaluations and will act as a pre-conditioner.

\section{Design Variables}

The design variable bounds for the upper and lower SCB geometry are illustrated in Table II. Table $\mathrm{V}$ shows design variable distribution for Hybrid-Game. It can be seen that the Nash-Players 1 and 2 consider only 3 design variables while the Global-Player of Hybrid-Game considers 6 design variables.

TABLE V

DESIGN VARIABLE DISTRIBUTION FOR HYBRID-GAME

\begin{tabular}{ccccc}
\hline \hline Type of & \multicolumn{3}{c}{ Hybrid-Game } & HAPMOEA \\
SCB & $G P$ & $N P 1$ & $N P 2$ & $($ Node $0 \sim 6)$ \\
\hline U_SCB & $\sqrt{ }$ & $\sqrt{ }$ & & $\sqrt{ }$ \\
L_SCB & $\sqrt{ }$ & & $\sqrt{ }$ & $\sqrt{ }$ \\
\hline \hline
\end{tabular}

\section{Implementation}

The following conditions are for Hybrid-Game; Global-Player, Nash-Player 1 and Nash-Player 2.

- GP: Population size of 10 with a grid of $36 \times 213$.

- NP1: Population size of 10 with a grid of $36 \times 213$.

- NP2: Population size of 10 with a grid of $36 \times 213$.

\section{Numerical Results}

As illustrated in Figure 10, the algorithm was allowed to run for 5 hours and 1,775 function evaluations using single $4 \times 2.8$ $\mathrm{GHz}$ processor. The algorithm converged at 683 function evaluations (approximately 1.9 hours) with $C d_{\text {Total }}=0.03437$ which HAPMOEA could not capture even after 24 hours shown in previous test Section B. To compare the computational efficiency of HAPMOEA and Hybrid-Game, the fitness value is chosen to $C d_{\text {Total }}=0.03441$ which HAPMOEA captured after 10 hours. The Hybrid-Game took 1.48 hours which is only $15 \%$ of HAPMOEA computational cost. In other words, Nash-Game improves performance of EA by $85 \%$ as shown in Figure 11. 


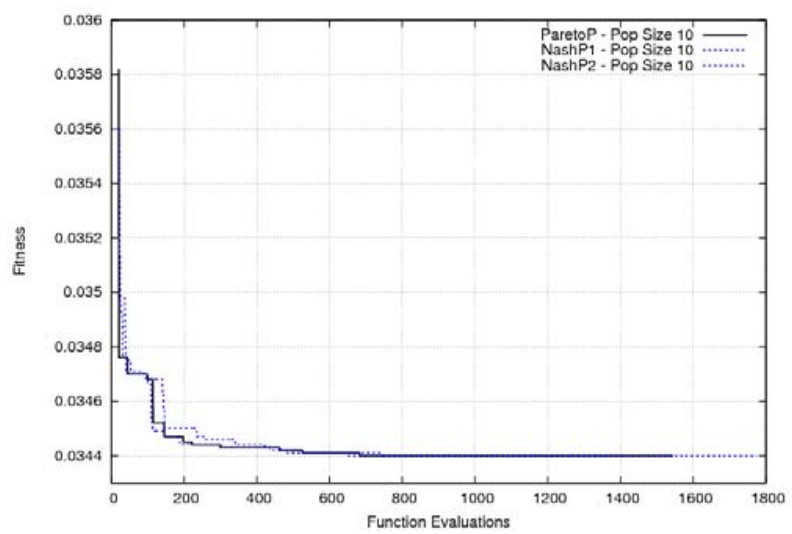

Fig. 10. Convergence objective using Hybrid-Game.

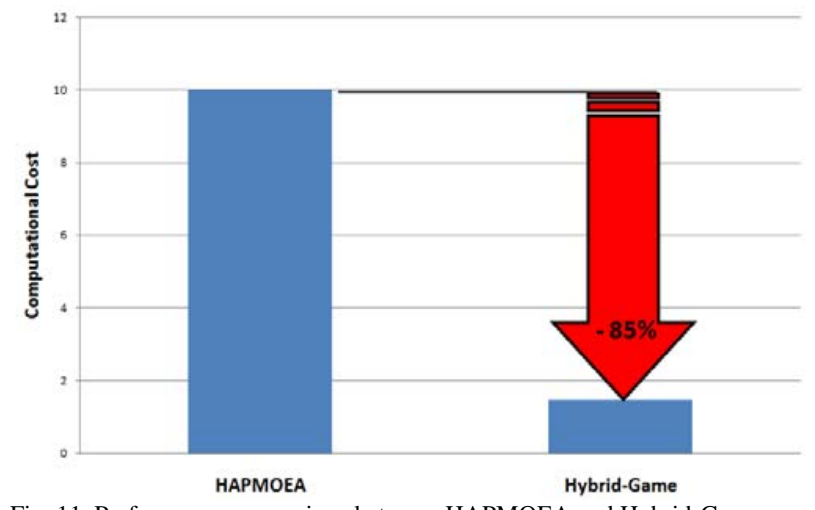

Fig. 11. Performance comparison between HAPMOEA and Hybrid-Game.

Table VI compares the aerodynamic characteristics obtained by the baseline design (RAE 5243) and the baseline design with upper SCB. Applying SCB on upper surface of RAE 5243 aerofoil saves the wave drag by $8 \%$ which leads $12 \%$ of total drag reduction. This optimal double SCB improves $L / D$ by $13.0 \%$.

TABLE VI

AERODYNAMIC CHARACTERISTICS

\begin{tabular}{|c|c|c|c|}
\hline Aerofoil & $C d_{\text {Total }}$ & $C d_{\text {Wave }}$ & $L / D$ \\
\hline Baseline & 0.03898 & 0.0088 & $\begin{array}{c}4.49 \\
509(+13 \%)\end{array}$ \\
\hline with SCB & $0.03437(-12 \%)$ & $0.0081(-8 \%)$ & $5.09(+13 \%)$ \\
\hline
\end{tabular}

Note: $\mathrm{Cl}$ is fixed to 0.175 .

The optimal double shape of double SCB obtained by Hybrid-Game is described in Table VII. It can be seen that the upper SCBs obtained by Hybrid-Game and HAPMOEA (Table IV) have almost same shape while the lower SCB from Hybrid-Game is $10 \%$ shorter than the lower SCB obtained by HAPMOEA.

TABLE VII

OPTIMAL SCB DESIGN VARIABLES OBTAINED BY HYBRID-GAME

\begin{tabular}{cccc}
\hline \hline Variables & Length $(\% c)$ & Height $(\% c)$ & Peak Position \\
\hline U_SCB & 23.65 & 0.649 & 84.99 \\
L_SCB & 23.88 & 0.384 & 80.35 \\
\hline \hline
\end{tabular}

Note: Peak position is in \% of SCB length. U_SCB and L_SCB represent SCB on the suction and pressure sides of RAE 5243 aerofoil. The U_SCB starts from $x$ and $y$ coordinates $(0.5067,0.0839)$ to $(0.7432,0.04774)$ and L_SCB position is located from $(0.4521,-0.0528)$ to $(0.6910,-0.0277)$.
Figure 12 illustrates the geometry of the baseline design and baseline with the optimal double SCB from HAPMOEA and Hybrid-Game. The baseline design with double SCB obtained by Hybrid-Game has same $t / c$ while the max camber is increased by 0.00055 and its position is moved $15 \%$ c towards to the trailing edge when compared to the baseline design.

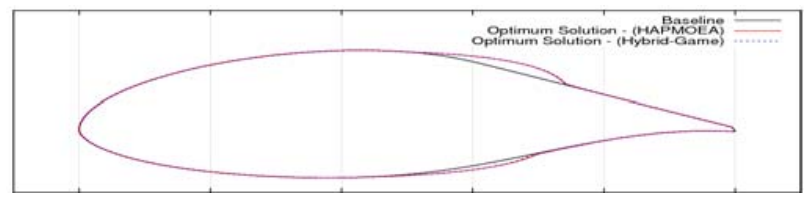

Fig. 12. Baseline design with the optimal double SCB (Note: $\max t / c=0.14$ at $41 \%$ c and max camber $=0.0214$ at $69.0 \%$ c).

Figure 13 shows the pressure contour of baseline design with the optimal double SCB obtained by Hybrid-Game. It can be seen that the upper shock is moved toward to the trailing edge while lower shock becomes weak isentropic waves.

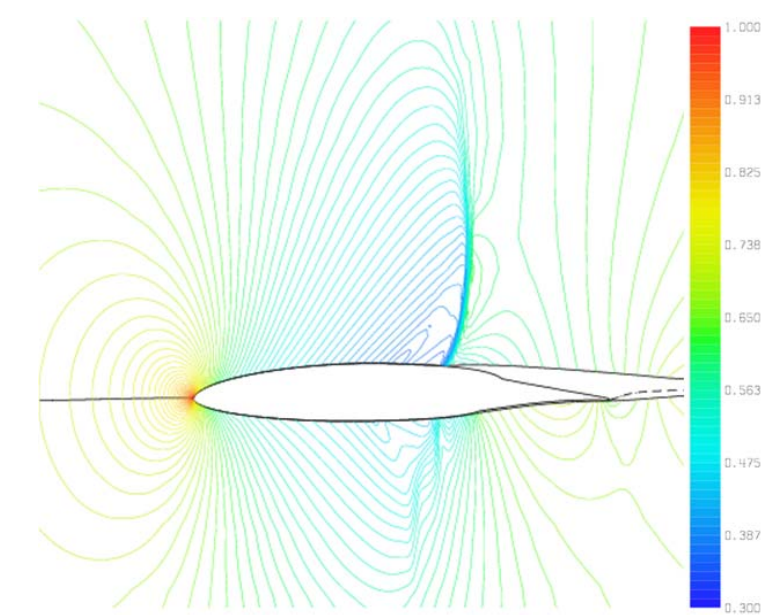

Fig. 13. $P / P_{0}$ contour of the optimal double SCB solution obtained by Hybrid-Game.

Figures $14 \mathrm{a})$ and $14 \mathrm{~b})$ compare a total drag $\left(C d_{\text {Total }}\right)$ and a wave drag $\left(C d_{\text {Wave }}\right)$ distributions obtained by the baseline design and with the optimal double SCB from both HAPMOEA and Hybrid-Game. The flow conditions are $M_{\infty} \in$ [0.5:0.85] with constant $C l_{\text {Fixed }}=0.175$ and $R e=18.63 \times 10^{6}$. It can be seen that both optimal double SCB obtained by HAPMOEA and Hybrid-Game perform almost same drag along the Mach numbers. The baseline design with the optimal double SCB starts to produce lower total drag when Mach number is higher than 0.71 . One thing should be noticed from Figure $14 \mathrm{~b})$ is that the critical Mach number $\left(M_{C}=0.65\right)$ for baseline design is extended to 0.71 by adding double SCB. The maximum total drag reduction (-26\%) is observed at $M_{\infty}=0.75$ shown in Figure 14 a) due to $88 \%$ of wave drag reduction shown in Figure $14 \mathrm{~b}$ ) when compared to the baseline design.

The baseline design with the optimal double SCB obtained by HAPMOEA and Hybrid-Game is also tested at five different flight conditions. The histogram showed in Figure 15 a) compares the total drag. It can be seen that the double SCB 
optimized at critical flight conditions reduces more total drag by 15 to $44 \%$ while improving the lift to drag ratio by 13.5 to $80 \%$ as shown in Figure 15 b) at the normal flight conditions.

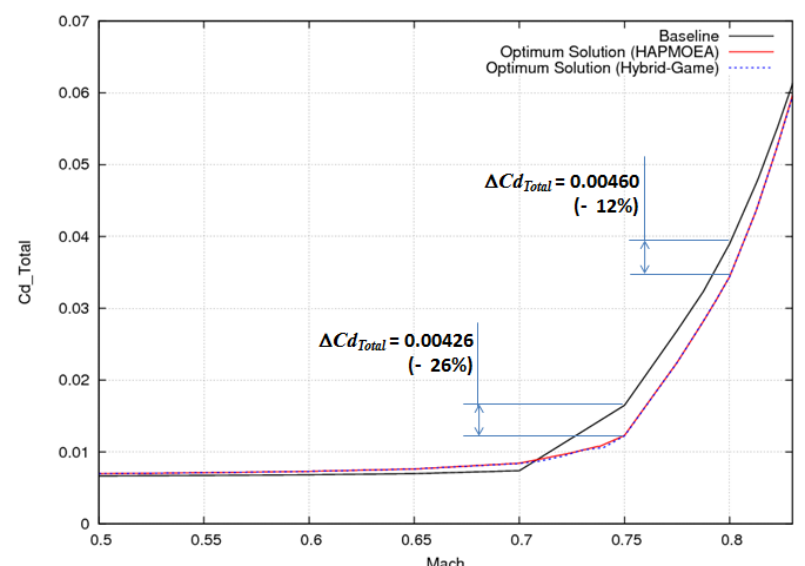

Fig. 14 a). $C d_{\text {Total }}$ vs. Mach numbers.



Fig. 14 b). $C d_{\text {Wave }}$ vs. Mach numbers.

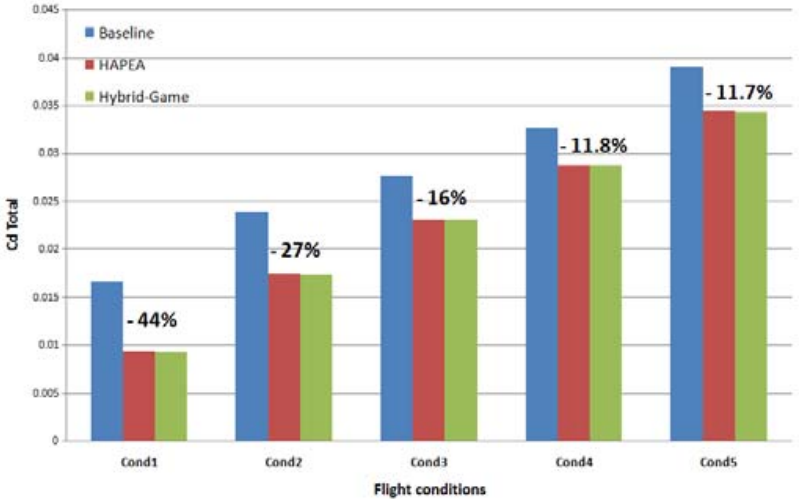

Fig. 15 a). Drag reduction obtained by the optimal double SCB at five different flight conditions.

Note: Cond $_{i}$ represents $i$ th flight conditions.

Cond $1: M_{\infty}=0.705, C l=0.690, R e=18.63 \times 10^{6}$

Cond $_{2}: M_{\infty}=0.730, C l=0.560, R e=18.63 \times 10^{6}$

Cond $3: M_{\infty}=0.750, C l=0.430, R e=18.63 \times 10^{6}$

Cond $_{4}: M_{\infty}=0.775, C l=0.300, R e=18.63 \times 10^{6}$

Cond ${ }_{5}: M_{\infty}=0.800, C l=0.175, R e=18.63 \times 10^{6}$

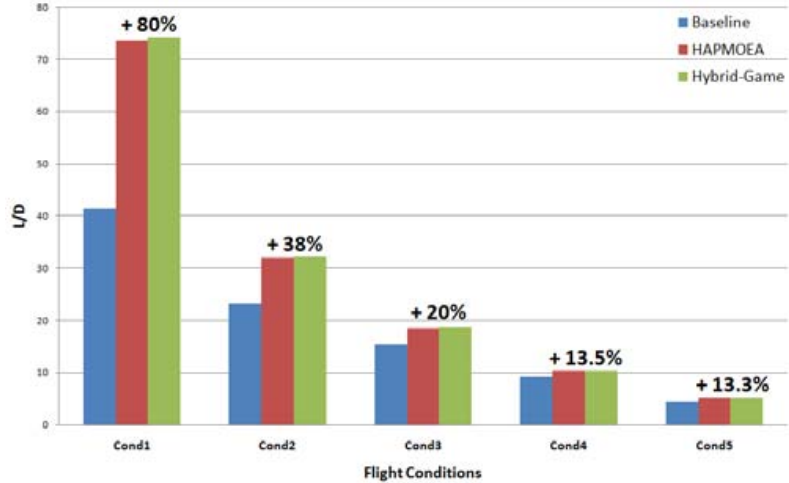

Fig. 15 b). $L / D$ obtained by the optimal double SCB at five different flight conditions.

One example $\left(\right.$ Cond $_{1}$ ) is shown in Figures 16 a) and 16 b) where the pressure ratio contours obtained by the baseline design and optimal solution from the Hybrid-Game are illustrated. Even though the double SCB is optimized at the critical flight condition, the optimal double SCB moves the normal strong shock shown in Figure 16 a) towards to the trailing edge by $10 \%$ c and reduce total drag by $44 \%$ which leads to $80 \%$ improvement of $L / D$.



Fig. 16 a). $P / P_{0}$ contour of the baseline design at Cond ${ }_{1}$ (Fig. 15a).

\section{RAE 5243 with Optimal Double SCB}

Fig. 16 b). $P / P_{0}$ contour of the optimal double SCB obtained by Hybrid-Game at Cond $_{1}$ (Fig. 15a). 
To summarise the optimisation test case, a double SCB on RAE 5243 is optimised using HAPMOEA and Hybrid-Game to reduce a transonic drag at the critical flight conditions. The use of optimal double SCB is beneficial at both normal and critical flow conditions. In addition, Hybrid-Game significantly reduces the computational cost for double SCB design optimisation while generating high quality optimal solution when compared to HAPMOEA.

The design engineer will choose the optimal double SCB obtained by Hybrid-Game which has $10 \%$ shorter length than SCB from HAPMOEA. In other words, the double SCB from Hybrid-Game will require less modification in current manufacturing system as well as less material.

\section{CONCLUSION}

In this paper, two advanced optimisation techniques have been demonstrated and implemented as a methodology for active flow control bump named Shock Control Bump shape design optimisation. Analytical research clearly shows the benefits of using Hybrid-Game in terms of computational cost and design quality. In addition, the use of SCB on current aerofoil reduces significantly transonic drag. In long term view, the use of SCB will save not only operating cost but also critical aircraft emissions due to less fuel burn.

Future work will focus on robust multi-objective design optimization of SCB (Taguchi method) which can produce the model with better performance and stability at variability of operating conditions and transition positions. In forthcoming research, other evolutionary optimiser including Strength Pareto Evolutionary Algorithm 2 (SPEA2), Self-adaptive Pareto Differential Evolution (SPDE) will be hybridized with Nash-Game strategy and their results will be compared in terms of solution quality and computational cost.

\section{ACKNOWLEDGMENT}

The authors gratefully acknowledge Dr. Eric J. Whitney, and Mourad Sefrioui, Dassault Aviation for fruitful discussions on Hierarchical EAs and their contribution to the optimisation procedure and, also to M. Drela at MIT for providing MSES software.

This work has been supported by the Spanish Ministerio de Ciencia e Innovación through project DPI2008-05250.

\section{REFERENCES}

[1] D. S. Lee, J. Periaux, and L. F. Gonzalez, UAS Mission Path Planning System (MPPS) Using Hybrid-Game Coupled To Multi-Objective Optimiser. Journal of Dynamic Systems, Measurement and Control, DS-09-1135. (In Press)

[2] K. Deb, S. Agrawal, A. Pratap, and T. Meyarivan. A fast and elitist multi-objective genetic algorithm: NSGA-II. IEEE Transactions on Evolutionary Computation, 6(2):182-197, 2002.

[3] D. S. Lee, L. F. Gonzalez, J. Periaux, and K. Srinivas, Hybrid-Game Strategies Coupled to Evolutionary Algorithms for Robust Multidisciplinary Design Optimization in Aerospace Engineering. IEEE Trans. Evolutionary Computation, TEVC-00213-2009. (In Press)

[4] J. Periaux, D. S. Lee, L. F. Gonzalez, and K. Srinivas, Fast Reconstruction of Aerodynamic Shapes using Evolutionary Algorithms and Virtual Nash
Strategies in a CFD Design Environment, Special Issue Journal of Computational and Applied Mathematics (JCAM). Vol. 232. Issue 1, pages 61-71, ISSN 0377-0427, 2009.

[5] D. S. Lee, L. F. Gonzalez, and E. J. Whitney, Multi-objective, Multidisciplinary Multi-fidelity Design tool: HAPMOEA - User Guide, Appendix-I, D.S. Lee, Uncertainty Based Multiobjective and Multidisciplinary Design Optimization in Aerospace Engineering, The Univ. of Sydney, Sydney, NSW, Australia. 2007.

[6] H. Bart-Smith, and P. E. Risseeuw, High Authority Morphing Structures, in Proceedings of IMECE 03, no. IMECE 2003-43377, (Washington, D.C), 2003 ASME International Mechanical Engineering Congress, November 2003.

[7] R. Oborn, S. Kota, and J. A. Hetrick, Active Flow Control Using High-Frequency Compliant Structures. Journal of Aircraft, Vol. 41, No. 3, pp 603-609. 2004.

[8] P. R. Ashill, L. J. Fulker, and A. Shires, A novel technique for controlling shock strength of laminar-flow aerofoil sections. Proceedings 1st Europian Forum on Laminar Flow Technology, pp. 175-183, Hamburg, Germany, DGLR, AAAF, RAeS, March 16-18 1992.

[9] N. Qin, Y. Zhu, and S. T. Shaw, Numerical Study of Active Shock Control for Transonic aerodynamics, International Journal of Numerical Methods for Heat \& Fluid Flow, Vol. 14 No. 4, pp 444 - 466, 2004.

[10] J. L. Fulker, and M. J. Simmons, An experiment study of shock control methods, DRA/AS/HWA/TR94007/1, Technical Report, DERA 1994.

[11] An Open Database Workshop for Multiphysics Software Validation, TA5: Shock Control Bump Optimisation On A Transonic Laminar Flow Airfoil. (Chairman N. Qin). Univ. Jyvaskyla, Finland, December 18, 2009 \& March 10-12, 2010.

[12] N. Hansen, A. Ostermeier, Completely Derandomized Self-Adaptation in Evolution Strategies. Evolutionary Computation, 9(2), pp. 159-195, 2001.

[13] N. Hansen, S.D. Müller, P. Koumoutsakos, Reducing the Time Complexity of the Derandomized Evolution Strategy with Covariance Matrix Adaptation (CMA-ES). Evolutionary Computation, 11(1), pp. 1-18, 2003.

[14] J. Wakunda, A. Zell, Median-selection for parallel steady-state evolution strategies. In Marc Schoenauer, Kalyanmoy Deb, Günter Rudolph, Xin Yao, Evelyne Lutton, Juan Julian Merelo, and Hans-Paul Schwefel, editors, ParallelProblem Solving from Nature - PPSN VI, pages 405-414, Berlin, Springer, 2000.

[15] D. A. Van Veldhuizen, J. B. Zydallis, G.B. Lamont, Considerations in Engineering Parallel Multiobjective Evolutionary Algorithms, IEEE Transactions on Evolutionary Computation, Vol. 7, No. 2, pp. 144-17, 2003.

[16] M. Sefrioui, J. Périaux, A Hierarchical Genetic Algorithm Using Multiple Models for Optimization. In M. Schoenauer, K. Deb, G. Rudolph, X. Yao, E. Lutton, J.J. Merelo and H.-P. Schwefel, editors, Parallel Problem Solving from Nature, PPSN VI, pages 879-888, Springer, 2000.

[17] D. S. Lee, Uncertainty Based Multiobjective and Multidisciplinary Design Optimization in Aerospace Engineering, The Univ. of Sydney, Sydney, NSW, Australia, Section 10.7, p.p. 348-370, 2008.

[18] M. Drela, A User's Guide to MSES 2.95. MIT Computational Aerospace Sciences Laboratory, September 1996.

[19] P. H. Cook, M. A. McDonald, M. C. P. Firmin, Aerofoil RAE 2822 Pressure Distributions, and Boundary Layer and Wake Measurements, Experimental Data Base for Computer Program Assessment, AGARD Report AR 138, 1979. 